\title{
RESEARCH ON NEW ENERGY GRID CONTROL TECHNOLOGY
}

\author{
LU Zhengtong, LI Shiqiang, WU Yingjun, SUN Lu, CHEN Guozhi, PENG Weilong \\ State Grid Zhoushan Power Supply Company \\ No.2-1, huiming bridge, dinghai district, zhoushan city, zhejiang province.
}

\begin{abstract}
Aiming at the poor waveform quality of the new energy grid connected under the traditional PI control strategy, based on the theory of repetitive control method, a compound control trategy is proposed. Through the analysis of the compound control module and simulation, the results show that grid harmonic distortion rate can be reduced about $2 \%$ at the same nonlinear load, the compound control strategy can suppress the load disturbance signal effectively, and the simulation results demonstrate the effectiveness of the proposed method.
\end{abstract}

\section{Introduction}

As the new energy technology is applied more and more widely, new energy grid can be efficient used became the focus of attention, so it is practical significance to improve the grid-connected technology.Inverter is the core device of the whole system grid-connected,inverter gridconnected control technology have directly affects on the power supply quality ${ }^{[1]}$. At present, the popular control methods include PI control strategy and fuzzy control, etc., but PI control strategy can't achieve the non-static tracking and cannot get a good results in a long time. Based on the limitation of PI control,in this paper proposes a compound control method based on repetitive control theory, the effectiveness of this strategy is verified through the analysis and demonstration of compound control strategy.

\section{Mathematical model of inverter.}

In order to facilitate the analysis, a single - phase inverter circuit is used to analyze the work characteristics.

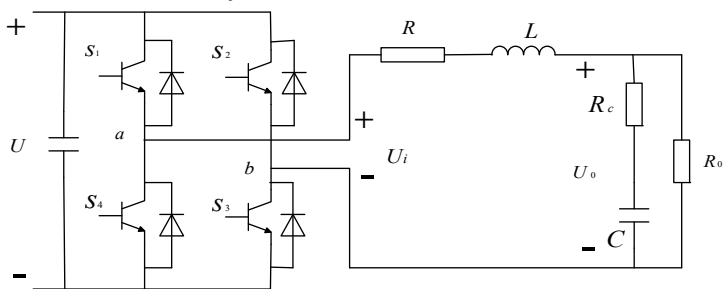

Figure1. Single-phase voltage source inverter

According to KVL and KCL law, the output current of inverter is as follows:

$$
i_{L}=i_{c}+i_{0}
$$

$$
U_{i}=U_{L}+R i_{L}+U_{0}=j \omega L+R i_{L}+U_{0}
$$

(Eq.2)
The output voltage of inverteris.: $U_{0}=R_{c} i_{c}+\frac{1}{j \omega C} i_{c}$

(Eq.3)

The output transfer function of inverter is :

$$
P(s)=\frac{U_{0}(s)}{U_{i}(s)}=\frac{\left(\frac{1}{s C}+R_{c}\right) / / R_{0}}{(s L+R)+\left(\frac{1}{s C+R_{c}}\right) / / R_{0}}
$$

(Eq.4)

From figure 1 knows that the inverter itself belongs to a nonlinear circuits and have a larger output impedance,the existence of the output impedance the same as the nonlinear circuit will distort the system, make the output current produces a series of spikes.

\section{The performance of inverter system under the PI control strategy.}

It is well known that voltage inverters are used to obtain the perfect current waveform, the PI control strategy is used commonly in most inverter control system. The following is a system performance of three-phase inverter grid under the double closed loop PI control strategy ${ }^{[2]}$, the relation between the three-phase voltage under the $\mathrm{dq}$ coordinate system as follows:

$$
\left\{\begin{array}{l}
U_{d}=-\left(k_{p}+\frac{k_{I}}{s}\right)\left(i_{d}{ }^{*}-i_{d}\right)-\omega L i_{q}+e_{d} \\
U_{q}=-\left(k_{p}+\frac{k_{I}}{s}\right)\left(i_{q}{ }^{*}-i_{q}\right)+\omega L i_{q}+e_{q}
\end{array}\right.
$$

In order to achieve the interconnection unit power factor, $I_{\mathrm{q}}{ }^{*}$ general was to be set 0 , so $i_{\mathrm{d}}{ }^{*}$ to be the key factor in the control circuit of the inverter.

Despite the current PI regulation can improve the steady state performance of the system, the compensation

\footnotetext{
${ }^{*}$ Corresponding author:e-mail: 1206434337@qq.com LU Zhengtong (phone: 13587053176; fax: 0580- 5111815)
} 
effect of this integral link is not obvious when a load mutation occurs, so the PI control can't eliminate the steady-state error of the system perfectly .

\section{Design of compound controller based on repetitive control theory.}

Repetitive control is based on the internal model which function is embedded the external signal model of system into controller ${ }^{[3]}$, there is a feedback control system which has good tracking ability and the ability to effectively eliminate disturbance, also is a control process to achieve zero error tracking too. According to the law of periodic disturbance, repetitive control has the same properties as grid harmonic signal in single cycle repeats. At this time, the following discrete internal model is:

$$
G(z)=\frac{Z^{-N}}{1-Z^{-N}}
$$

$\mathrm{T}$ is the system sampling period, $\mathrm{T}_{0}$ is the network voltage base wave period, $\mathrm{f}_{0}=50 \mathrm{~Hz}$, and $\mathrm{N}$ is the sampling number of the base wave period.

Due to internal model have characteristic of single cycle time delay, so the strategy have the results that system can't tracking and regulating to input signal timely when the reference signal mutation cycle, ${ }^{[4]}$, it means the system dynamic performance is poorer.

Since repetitive control has the characteristics of single cycle delay, the improvement structure is generally adopted as follow. The improvement structure is compound control which combine the repetitive control with PI control strategy, the following is the structure diagram of the compound control system.

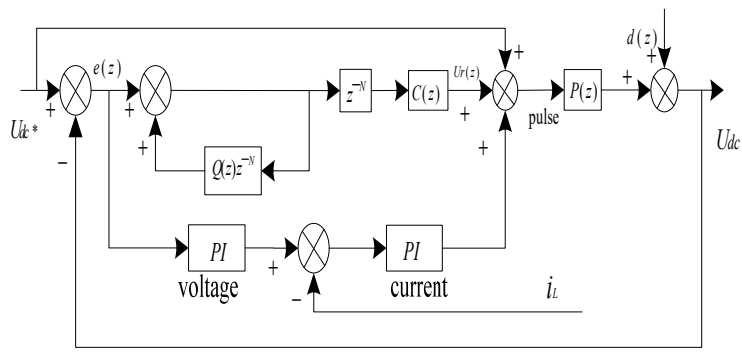

Figure 2.Single-phase inverter compound control system diagram

As is shown in Figure 2, the repetitive control strategy adopts the embedded structure where $d(\mathrm{z})$ is the interference signal, $i_{L}$ is the ac side current of inverter, and $P(\mathrm{z})$ is the output transfer function of the inverter system. Thanks to PI control strategy and repetitive control strategy work independently, so the combination of repetitive control with PI control can realize astatic tracking to the system, the system can get a good dynamic response performance and inhibition to jamming signal .

\section{Design of repetitive control structure parameters.}

In order to reflect the superior performance of repetitive control ${ }^{[6]}$, the following parameters are designed to be carried out in the no-load of the inverter. The inverter has the maximum harmonic distortion and minimum damping in no-load operation. The sampling frequency is $10 \mathrm{kH}_{\mathrm{Z}}$ in system, the output capacitance of inverter $\mathrm{C}$ is $200 \mathrm{uF}$, inductance $\mathrm{L}$ is $1 \mathrm{mH}$ and equivalent resistance is $1 \Omega$, the resonance frequency of systemis $6742.2 \mathrm{rad} / \mathrm{s}$ and damping ratio is 0.0405 .

Therefore, the no-load transfer function of the inverter system is:

$$
P(s)=\frac{45454564}{s^{2}+546 s+45454564}
$$

It can be seen from Eq. 8 konws that the controlled object contains high frequency resonance peak and has a certain phase lag too.

Thanks to the controlled object has the above properties, the model of elimination is proposed here. ${ }^{[7]}$, there have a compensation part $C(\mathrm{z})=\mathrm{K}_{\mathrm{r}} \mathrm{z}^{\mathrm{k}} \mathrm{S}_{2}(\mathrm{z}) \mathrm{S}_{1}(\mathrm{z})$ to be designed in the repetitive control. Thanks to the controlled object have dead zone effect and nonlinear load harmonic is primarily in the low frequency, $S_{2}(\mathrm{z})$ is a notch filter which is used to offset the resonance peak of the controlled object. $\mathrm{S}_{1}(\mathrm{z})$ is a low pass filter used to attenuate the high frequency harmonic signal after the resonant peak $Z^{\mathrm{k}}$. For phase advance, the phase lag is compensated by a certain phase lag between the filter $S_{1}$ and the no-load controlled object $P(\mathrm{~s})$.

The general expression of notch filter is:

$$
S_{2}(z)=\frac{z^{m}+a+z^{-m}}{2+a}
$$

Due to $z=e^{j \omega T}=e^{j \theta}$,

$$
S_{2}(\theta)=\frac{2 \cos m \theta+a}{2+a}
$$

When $S_{2}(\theta)$ is equal to 0 , the trap has the strongest attenuation of a particular frequency.It can be obtained the relation is $2 \cos m \theta+a=0$, when and only if $a=2$ meets the conditions. At this time, there have the relation $\mathrm{m} \theta=\pi$ and $\theta=\mathrm{wT}$, so we can get the result $\mathrm{m}=\pi / \theta=3.58$, the value of $\mathrm{m}$ is defined to 4 in here. then the trap wave filter at this time is:

$$
S_{2}(z)=\frac{z^{4}+2+z^{-4}}{4}
$$

The following is the low-pass filter model of $S_{1}(\mathrm{z})$, the model of the second-order low-pass filter is:

$$
S_{1}(z)=\frac{\omega_{n}{ }^{2}}{s^{2}+2 \zeta \omega_{n} s+\omega_{n}{ }^{2}}
$$

According to the parameters of this system:

$$
S_{1}(z)=\frac{0.1813 z+0.1551}{z^{2}-1.094 z+0.33}
$$

From the above analysis knows that both the secondorder low-pass filter $S_{1}$ and the no-load controlled object $P(\mathrm{~s})$ have certain phase $\operatorname{lag}^{[8]}$. In order to maintain the stability of the system, the phase advance link $\mathrm{z}^{\mathrm{k}}$ is designed to here. Designed by the above relationship 
between trap and second-order filter can launch the beat the relationship between $\mathrm{k}$ and frequency in advance, the research shows that when $\mathrm{k}$ is available between 4 to 5 best compensation effect, the advanced beat is 5 in this paper, namely the $z^{k}=z^{5}$.

\section{The simulation of system under the compound control strategy.}

Three-phase inverter compound control system as shown in figure 3, The dc reference voltage $U_{\mathrm{dc}}{ }^{*}$ is $660 \mathrm{~V}$, the power grid voltage is $380 \mathrm{~V}$, filtering inductance capacitance $L$ is $1.5 \mathrm{mH}$ and $\mathrm{C}$ is $200 u \mathrm{~F}$, the output equivalent resistance $\mathrm{R}$ is $1 \Omega$, the PI control parameters of current loop for the $\mathrm{K}_{\mathrm{p}}$ is 5 and $\mathrm{K}_{\mathrm{i}}$ is 66, the PI parameters of voltage loop for $\mathrm{K}_{\mathrm{p}}$ is 2.1 and $\mathrm{K}_{\mathrm{i}}$ is 1000 . The switching frequency $f$ is $10 \mathrm{kHz}$, the repeated control module parameters are consistent with the above.

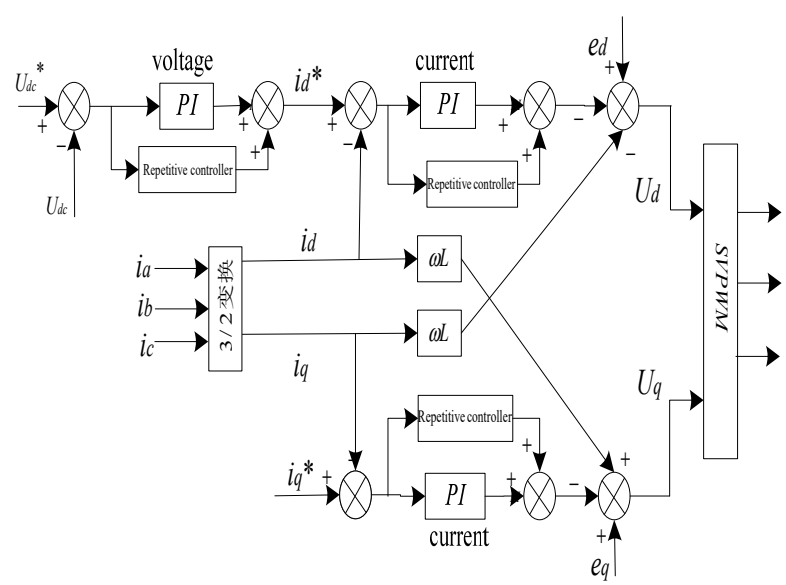

Figure 3 .Three-phase inverter system simulation diagram of composite control structure

(1) The simulation results of nonlinear load under double closed loop PI control and compound control strategy.

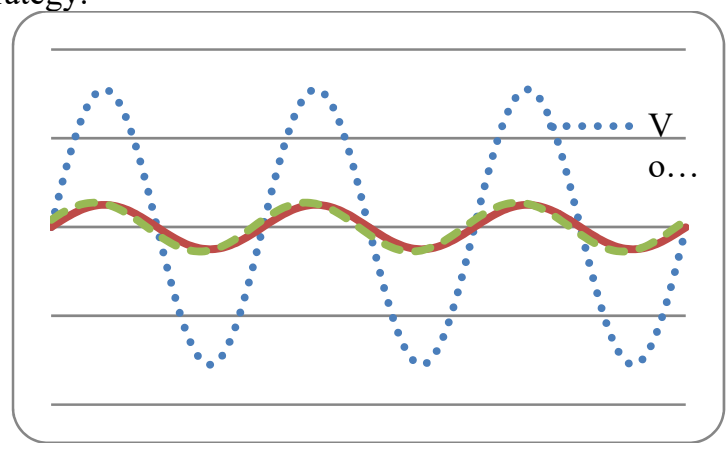

Figure 4. The grid voltage and current waveform

Figure 4 shows the voltage and current waveform of the parallel network, the voltage unit is $\mathrm{V}$ and current unit is $\mathrm{A}$ in all the graphs. Current 1 is the current waveform under compound control strategy of the grid-connected, and the current 2 is the waveform under PI control strategy of grid-connected.The results shows that the harmonic distortion rate of the grid-connected current 2 is $6.83 \%$ and the current 1 is $4.31 \%$ at the same load condition,

(2) The following is the grid-connected simulation of the compound control under voltage fluctuation of the power grid.

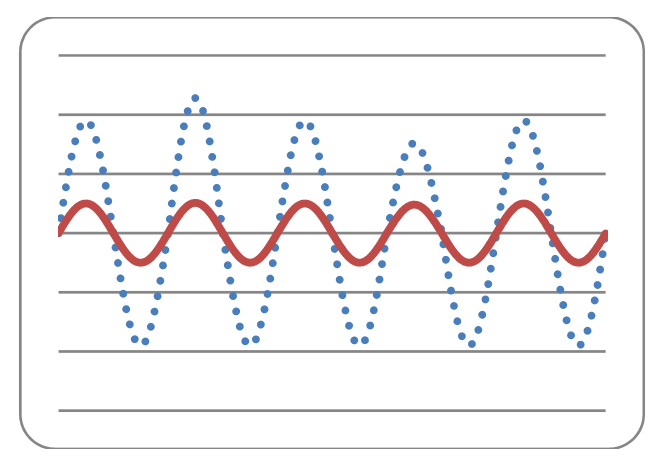

Figure 5. The grid current waveform under compound control

The simulation results show that the harmonic distortion rate of the compound control is reduced from $6.83 \%$ to $4.31 \%$ at the same nonlinear load, the voltage of the power network increased by $20 \%$ from $0.02 \mathrm{~s}$ to $0.03 \mathrm{~s}$ and reduced by $20 \%$ from $0.06 \mathrm{~s}$ to $0.07 \mathrm{~s}$. The time of both fluctuations was $0.01 \mathrm{~s}$, and the network current was basically stable without any obvious fluctuation.

\section{7 conclusion}

The proposed compound control strategy is based on repetitive control theory, according to the results of analysis and simulation we knows that the control strategy can effectively reduce the grid current harmonic distortion rate and the load fluctuation signal can be suppressed effectively, the steady-state accuracy of the system has been improved effectively under the strategy.

\section{Acknowledgment}

Fund project: The paper was funded by the state grid corporation science and technology project.The project name is research on new energy electricity generation foundation multivoltage classes direct current collection network organization and operational control technology ,the project number is $5211 \mathrm{ZS} 1600 \mathrm{NP}$.

\section{REFERENCES}

1. Lu Sun;A Survey of Repetitive Control for Nonlinear Systems. -Science Foundation in China 3:15-16.

2. Wenli Sun; Hong Cai; Fu Zhao: FBFN-based adaptive repetitive control of nonlinearly parameterized systems. -Journal of Systems Engineering and Electronics 5:115-116. 
3. Edi Kurniawan; Zhen-Wei Cao; Maria Mitrevska; Zhi-Hong Man: Design of Decentralized Multi-input Multi-output Repetitive Control Systems. International Journal of Automation and Computing 12:75-76.

4. Quan Quan; Kai-Yuan Cai: Repetitive Control for TORA Benchmark:An Additive-statedecomposition-based Approach. -International Journal of Automation and Computing 18:45-46.

5. Wen-Long Ming,Qing-Chang Zhong;A Single-Phase Rectifier Having Two Independent Voltage Outputs With Reduced Fundamental Frequency Voltage Ripples[J].IEEE Transactions on Power Electronics,2015,30(11):3662-3673.
6. Bingxi Jia, Shan Liu , Yi Liu;Visual trajectory tracking of industrial manipulator with iterative learning control[J].Industrial Robot,2015,42(78):1213.

7. Zhang Kai,Kang Yong,Xiong Jian;Study on an inverter with pole assignment and repetitive control for UPS applications[J]. Proceedings of PIEMC,2012,23(2):45-48.

8. Andersen B R;HVDC Transmission-Opportunities and Challenges[C].The $8^{\#}$ IEEE International Conference on AC and DC Power Transmission,2831March 2006,24-29. 